\title{
Presence of lysogenic phage in the outbreak strains of Vibrio cholerae 0139
}

\author{
S. N. MITRA, S. KAR, R. K. GHOSH*, S. PAJNI $\dagger$ and A. GHOSH $\dagger$ \\ Indian Institute of Chemical Biology, 4 Raja S.C. Mullick Road, Jadavpur, Calcutta-700 032 and $\uparrow$ Institute of \\ Microbial Technology, Sector 39-A, Chandigarh-160 014, India
}

\begin{abstract}
Summary. Four outbreak strains of Vibrio cholerae O139 from endemic areas of India and Bangladesh were found to carry lysogenic phage(s). All of these phage(s) produced turbid plaques characteristic of lysogeny on $V$. cholerae MAK 757 (El Tor, Ogawa) cells as well as on their VcA-1 lysogens but were unable to infect $V$. cholerae 154 (classical) cells, the universal host for all classical phages. Colonies in the turbid plaques were O139 lysogens and these developed an auxotrophic requirement, mainly for purines suggesting the integration of the prophage into the host chromosome. The immunity profile of the 0139 phage(s) was similar to that of phage $\alpha$ but differed in the sensitivity of the phage lysogen of $V$. cholerae MAK 757 to subsequent infection by phage $\beta$.
\end{abstract}

\section{Introduction}

Vibrio cholerae serogroup $\mathrm{O} 1$, the causative agent of cholera, has been responsible for seven major pandemics since $1814 .^{1}$ In contrast, $V$. cholerae serogroup non-O1 strains are associated only with sporadic cases of gastro-enteritis and extra-intestinal infection but lack endemic potential. ${ }^{2}$ However, since October, 1992, a typical cholera-like epidemic has broken out in India and Bangladesh and swiftly assumed endemic proportions, involving thousands of people with significant mortality. ${ }^{3}$ The causative agent was identified as a $V$. cholerae strain which was serologically different from hitherto known serogroups. The outbreak strains isolated from various endemic areas of India and Bangladesh were serologically identical and were assigned a new serogroup called O139 with a synonym "Bengal"." All O139 strains isolated so far produce cholera toxin (CT), hybridise with a CT gene probe ${ }^{5}$ and at least four of the strains examined carry an unusual number of tandem repetitions of the toxin gene on their chromosomes. ${ }^{6}$ The swift spread of this O139 serogroup coupled with its propensity to displace the existing pandemic serogroup O1 El Tor strains, appears to mark the beginning of a new pandemic. The genesis of this novel strain of $V$. cholerae has evoked global interest and concern. Whether these new strains are mutants of serogroup $\mathrm{Ol}$ or have originated from

Received 29 Dec. 1993; revised version received 12 Oct. 1994; accepted 1 Dec. 1994

* Correspondence should be sent to Dr R. K. Ghosh.
non-O1 strains which have acquired copies of the toxin gene, is a matter of debate. Bacteriophages, because of their strict host specificity, can be a very useful tool for examining this question. In this study, O139 strains were examined for lysogenic bacteriophage(s).

\section{Materials and methods}

\section{Bacteria and bacteriophages}

Fourteen $V$. cholerae 0139 strains from endemic areas of India and Bangladesh were examined; they comprised two each from Madras (strains MO 1, MO 28), Andhra Pradesh (AP 1, AP 2), Vellore (VO 15, VO 20), Madurai (MDO 12, MDO 25), Calcutta (SG 24, SG 25), Amravati (PO 2, PO 7) and Bangladesh (BO 2, BO 4). These $\mathrm{O} 139$ strains and $V$. cholerae non-O1 $\mathrm{CT}^{-}$strains were kindly provided by $\mathrm{Dr}$ G. B. Nair, National Institute of Cholera and Enteric Diseases, Calcutta. V. cholerae, classical Ogawa strain 154 was from Dr S. Mukherjee of this Institute. The test strains were maintained on nutrient-agar slopes and subcultured at 2-week intervals. Choleraphage $149, \mathrm{e}_{4}$ and temperate phages $\alpha, \beta$ and $\mathrm{VcA}-1$ were obtained from Dr F. K. Bhattacharya.

\section{Media and growth conditions}

$V$. cholerae strains were grown in nutrient broth containing Bactopeptone (Difco) 1\%, Lablemco 
powder (Oxoid) $1 \%, \mathrm{NaCl} 0 \cdot 5 \%$ at $37^{\circ} \mathrm{C}$ with shaking $(180 \mathrm{rpm})$. Addition of Bactoagar (Difco) $2 \%$ to the nutrient broth constituted the nutrient agar medium. To determine the sensitivity of $V$. cholerae to phages, logarithmic-phase cells of the host bacteria on nutrient agar were overlayed with soft agar (nutrient broth containing agar $0.8 \%$ ). Suitable dilutions of the phage preparations were then spotted on the plates. Results were read after incubation for $12 \mathrm{~h}$ at $37^{\circ} \mathrm{C}$. V. cholerae strains classical 154 and El Tor MAK 757 were used as indicator hosts for the phages obtained from $V$. cholerae $\mathrm{O} 139$ strains. $^{7}$

\section{Prophage induction}

Mitomycin C. V. cholerae $\mathrm{O} 139$ strains containing phage were grown as described above at $37^{\circ} \mathrm{C}$ to an absorbance at $540 \mathrm{~nm}\left(\mathrm{~A}_{540}\right)$ of 0.2 . The cells were collected by centrifugation, washed with saline $0.9 \%$ to remove free phage and then suspended in saline. The suspension was divided into aliquots to which mitomycin $\mathrm{C}$ was added at increasing concentrations up to $2.5 \mathrm{mg} / \mathrm{L}$. After incubation for $10 \mathrm{~min}$ at $37^{\circ} \mathrm{C}$, the mitomycin $\mathrm{C}$ was removed by centrifugation; the cells were washed with saline suspended in nutrient broth and incubated at $37^{\circ} \mathrm{C}$. Samples were withdrawn at various intervals, treated with drops of chloroform and centrifuged. Supernates were assayed for phage after appropriate dilution.

Ultraviolet radiation. $V$. cholerae 0139 strains containing phage were grown up to $A_{540}$ of 0.2 as above and collected by centrifugation. The cells were suspended in saline $\left(2 \times 10^{8}\right.$ cells $\left./ \mathrm{ml}\right)$ and distributed in small volumes on petri dishes to form a thin layer. Each sample was irradiated for 2 min with a $15 \mathrm{~W}$ Philips germicidal lamp, specifically emitting radiation of $254 \mathrm{~nm}$ wavelength, held $50 \mathrm{~cm}$ above the plate. The samples were collected, transferred to nutrient broth and incubated at $37^{\circ} \mathrm{C}$ for $6 \mathrm{~h}$. The cells were assayed for phage. Un-irradiated cells were used as controls.

\section{Test for auxotrophy}

Lysogens of $V$. cholerae MAK 757 with 0139 phage were grown on nutrient agar plates to give single colonies. These were then replicated on to 12 minimal agar plates supplemented with 12 different combinations of nutrients (amino acids, vitamins, purines, pyrimidines). ${ }^{8}$ Plates were incubated overnight at $37^{\circ} \mathrm{C}$ and auxotrophy was determined by comparing the relative growth pattern on plates containing different nutrient pools.

\section{Serotyping}

Lysogens of $V$. cholerae MAK 757 with 0139 phage were serotyped by the slide agglutination procedure described by Siddiqui and Bhattacharya ${ }^{9}$ with appropriate dilutions of $\mathrm{O} 1$ and $\mathrm{O} 139$ antisera (kindly provided by Dr G. B. Nair). Colonies showing immediate agglutination were read as positive.

\section{Isolation of phage(s) from 0139 strains}

Strains were grown in nutrient broth overnight at $37^{\circ} \mathrm{C}$ with shaking. Free phages liberated by these strains were collected by removing the cells by centrifugation at $10000 \mathrm{~g}$ for $10 \mathrm{~min}$. The preparation was then treated with chloroform to kill any contaminating bacteria.

\section{Results}

\section{Liberation of phage by 0139 strains}

$V$. cholerae 0139 strains isolated from various endemic areas were grown overnight and the culture supernates were collected for identification of the phage. To confirm that these preparations did not contain any cells, the supernates were streaked on nutrient agar plates and incubated overnight at $37^{\circ} \mathrm{C}$. All the preparations were free of viable bacteria. In the soft agar overlay assay with $V$. cholerae El Tor MAK 757 and classical 154, supernates of O139 strains from Calcutta (SG 25), Bangladesh (BO 2), Amravati (PO 7) and Madurai (MDO 25) produced turbid plaques characteristic of lysogenic phage of $V$. cholerae MAK 757 (figure). To confirm that the colonies inside the plaques were true lysogens, growth was picked from the centre of the plaques and streaked for single colonies, which were then subcultured 10 times and streaked for single colony isolation each time to remove any contaminating phages. The final subcultures were then treated to liberate free phages and examined for susceptibility to 0139 phages. All the colonies examined liberated free phage and were

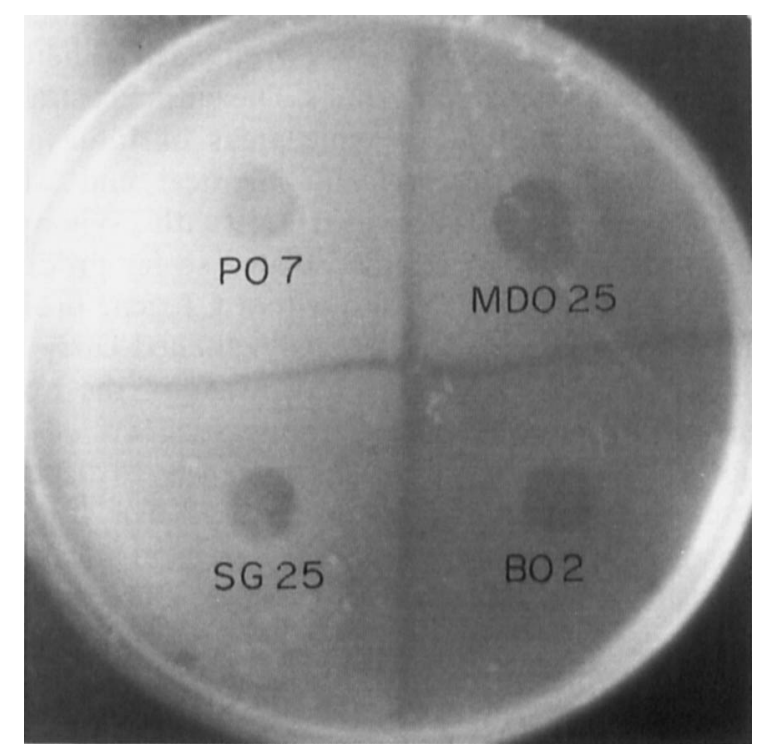

Figure. Sensitivity of $V$. cholerae MAK 757 El Tor Ogawa to the phages liberated from O139 strains from Calcutta (SG 25), Bangladesh (BO 2), Amaravati (PO 7) and Madurai (MDO 25). 
Table I. Susceptibility of $V$. cholerae 154 and MAK 757 and MAK 757 lysogens to O139 phage

\begin{tabular}{|c|c|c|c|c|c|c|c|}
\hline \multirow{2}{*}{ Strains } & \multicolumn{7}{|c|}{ Susceptible to infection with phage } \\
\hline & $\alpha$ & $\beta$ & VcA-1 & BO 2 & PO 7 & MDO 25 & SG 25 \\
\hline \multicolumn{8}{|l|}{ Parental } \\
\hline V. cholerae MAK 757 & + & + & + & + & + & + & + \\
\hline$V$. cholerae 154 & - & - & - & - & - & - & - \\
\hline \multicolumn{8}{|l|}{ Lysogens } \\
\hline MAK $757(\alpha)$ & - & + & + & - & - & - & - \\
\hline MAK $757(\beta)$ & - & - & + & - & - & - & - \\
\hline MAK 757 (VcA-1) & + & + & - & + & + & + & + \\
\hline MAK 757 (BO 2) & - & - & - & - & - & - & - \\
\hline MAK 757 (PO 7) & - & - & - & - & - & - & - \\
\hline MAK 757 (MDO 25) & - & - & - & - & - & - & - \\
\hline MAK 757 (SG 25) & - & - & - & - & - & - & - \\
\hline
\end{tabular}

Table II. Host-range of phages from $V$. cholerae $\mathrm{O} 139$ strains

\begin{tabular}{|c|c|c|c|c|}
\hline \multirow{2}{*}{ Host } & \multicolumn{4}{|c|}{$\begin{array}{l}\text { Susceptible to infection with } \\
\text { phages from strain }\end{array}$} \\
\hline & SG 25 & PO 7 & MDO 25 & $\mathrm{BO} 2$ \\
\hline \multicolumn{5}{|l|}{$V$. cholerae $\mathrm{O} 139$ strains } \\
\hline $\begin{array}{l}\text { MO } 1, \text { MO } 28, \text { AP } 1, \\
\text { AP } 2, \text { VO } 15, \text { VO } 20 \\
\text { MDO } 12, \text { MDO } 25 \\
\text { SG } 24, \text { SG } 25, \text { PO } 2 \\
\text { PO } 7, \text { BO } 2, \text { BO } 4\end{array}$ & - & - & - & - \\
\hline $\begin{array}{l}\text { V. cholerae MAK } 757 \\
\text { El Tor, Ogawa }\end{array}$ & + & + & + & + \\
\hline $\begin{array}{l}\text { V. cholerae MAK } 757 \\
\text { (VcA-1) }\end{array}$ & + & + & + & + \\
\hline $\begin{array}{l}V . \text { cholerae } 154 \\
\text { classical, Ogawa }\end{array}$ & - & - & - & - \\
\hline \multicolumn{5}{|l|}{$\begin{array}{l}\text { V. cholerae Non-O1 } \\
\text { CT }^{-}\end{array}$} \\
\hline $\begin{array}{l}\text { VCE } 50, \text { VCE } 20 \\
\text { VCE } 49\end{array}$ & - & - & - & - \\
\hline
\end{tabular}

immune to further infection by the same phage preparation (table I).

\section{Host range of the 0139 phage}

In soft-agar overlay assays with $V$. cholerae indicator strain of various bio- and serotypes, all of the O139 phage preparations produced turbid plaques on $V$. cholerae El Tor MAK 757 but were unable to infect $V$. cholerae classical 154, various environmental $V$. cholerae non-O1 $\mathrm{CT}^{-}$strains or the $V$. cholerae $\mathrm{O} 139$ strains (table II).

\section{Immunity pattern of the O139 phage}

Temperate phages of $V$. cholerae, belong to three known immunity classes- $\kappa$ and $\beta^{10}$ and VcA-1. ${ }^{11}$ To determine whether the $\mathrm{O} 139$ phage(s) belong to any of these immunity classes, advantage was taken of the different ability of the three classes of lysogens to infect reciprocal lysogens of $V$. cholerae MAK 757. $V$. cholerae MAK 757 cells were lysogenised with VcA-1, $\beta$ and $\alpha$ ( $\kappa$ class) phages, ${ }^{12}$ as well as with the O139 phages and the resulting lysogens were compared for their sensitivity to infection by each of the phages. Phages from all O139 strains resembled phage $\alpha$ in their ability to infect $V$. cholerae MAK 757 as well as its VcA-1 lysogen and their inability to infect $V$. cholerae classical strains and $\alpha$ and $\beta$ lysogens of $V$. cholerae MAK 757, but they differed from phage $\alpha$ in that $\alpha$ lysogens of $V$. cholerae MAK 757 are sensitive to $\beta$ phage infection whereas $V$. cholerae MAK 757 lysogenised with $\mathrm{O} 139$ phage was immune to $\beta$ phage infection (table I). All the $V$. cholerae 0139 strains were immune to infection by $\alpha, \beta$ and VcA-1 phages. $V$. cholerae MAK 757 lysogens of $\mathrm{O} 139$ phages were resistant to super-infection by $\mathrm{O} 139$ phages.

\section{Prophage induction by ultraviolet light and mitomycin $C$}

None of the $V$. cholerae 0139 cells carrying the phage or the $V$. cholerae MAK 757 lysogens of O139 phage could be induced to lyse, and show any increases in phage titre by treatment with UV light or mitomycin $\mathrm{C}$.

\section{Auxotrophy}

Because integration of the prophage in the chromosome is likely to inactivate genes and thereby induce auxotrophy at the site of integration, the original host strains and the putative lysogens were plated on minimal agar plates supplemented with amino acids, nitrogenous bases (purines and pyrimidines) or vitamins. Of the four $V$. cholerae $\mathrm{O} 139$ strains carrying the phase, only strain BO 2 required purines for their growth on minimal media; all the other $\mathrm{O} 139$ strains were prototrophs. However, $c .75 \%$ of the $V$. cholerae MAK 757 lysogens had acquired a requirement for purines (table III).

\section{Serotyping of $V$. cholerae $M A K 757$ lysogens}

To determine whether lysogenisation by $\mathrm{O} 139$ phage plays any role in serotype conversion to generate the endemic $\mathrm{O} 139$ strain, a large number of $V$. cholerae 
Table III. $V$. cholerae O139 phage-induced auxotrophies in lysogenised $V$. cholerae MAK 757 and $V$. cholerae $\mathrm{O} 139$ cells

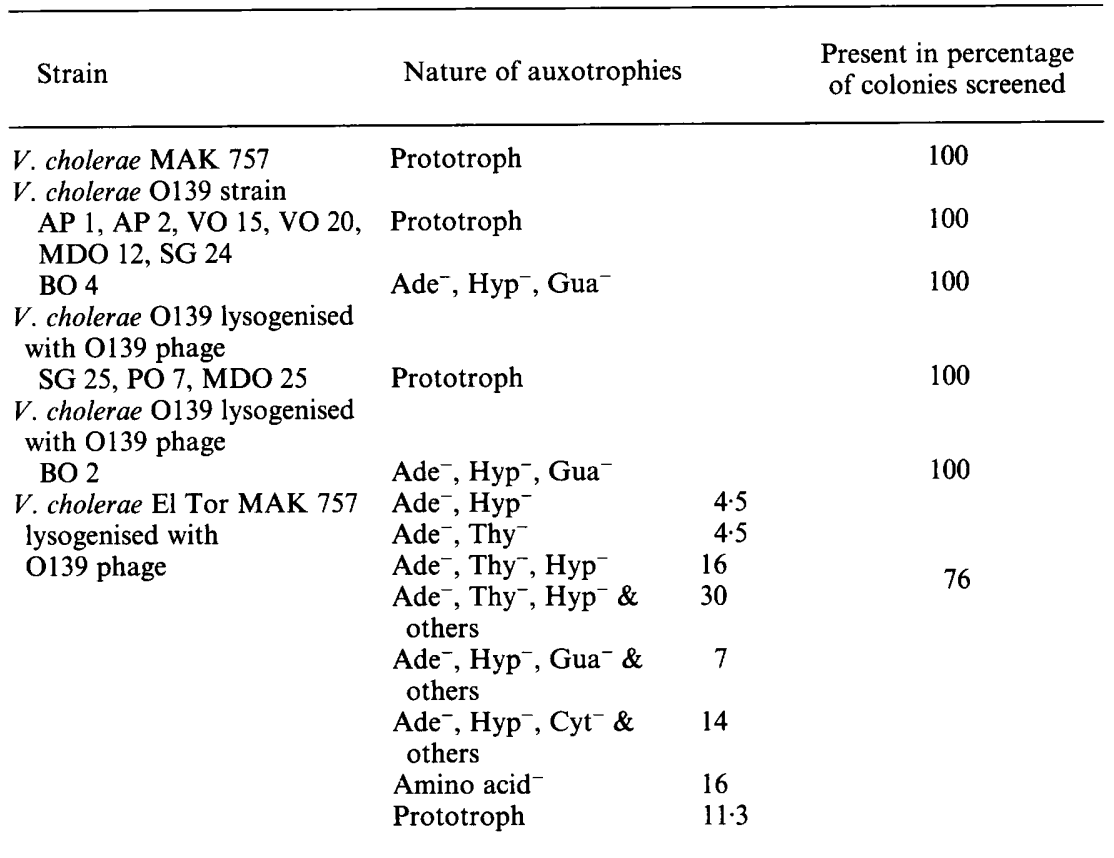

Each experiment involved analysis of 1000 single colony isolates. The experiment was repeated five times. Ade, adenine; Cyt, cytosine; Gua, guanosine; Hyp, hypoxanthine; Thy, thymine.

MAK 757 lysogens of O139 phage were serotyped with polyclonal $\mathrm{O} 1$ and $\mathrm{O} 139$ antisera. None of the lysogens acquired the O139 serotype; all maintained their parental O1 serotype.

\section{Discussion}

The emergence of $V$. cholerae $\mathrm{O} 139$ as a new strain causing endemic cholera and replacing the existing biotype has received global attention. The strain has been characterised serologically and is being examined at biochemical and molecular levels. Recently we presented evidence that the strain emerged from serogroup $\mathrm{O} 1$ strains and possible progenitor strains have been identified. ${ }^{13}$ The present report describes the detection of phage(s) in some O139 strains. The slow release of phage in broth culture, even after repeated subculture followed by single colony isolation, the immunity of the parent strain to further infection by the same phage, the formation of turbid plaques on $V$. cholerae MAK 757 and induction of auxotrophy in this host indicate that this is a temperate phage. However, treatment with UV irradiation or mitomycin $\mathrm{C}$ failed to induce lysis in any of the $\mathrm{O} 139$ strains or in the $V$. cholerae MAK 757 lysogens. A similar property has also been reported for other temperate phages of $V$. cholerae, namely phages CP-T $1{ }^{14}$ and PS166 (S. N. Mitra and R. K. Ghosh, unpublished observation). The 0139 phage could infect $V$. cholerae MAK 757 (El Tor, Ogawa), the universal host for all El Tor phages, ${ }^{7}$ but not $V$. cholerae 154 (classical, Ogawa), the universal host for all classical phages. ${ }^{7}$ As bacterio- phages usually maintain strict host specificity, the results were indicative that $\mathrm{O} 139$ strains are more closely related to the El Tor than to the classical strains of $V$. cholerae. This observation further strengthens and extends our conjecture that $\mathrm{O} 139$ strains arose from the progenitor strains which themselves evolved from the $V$. cholerae $\mathrm{O} 1 \mathrm{El}$ Tor strains by mutation. ${ }^{13}$

All the $V$. cholerae $\mathrm{O} 139$ strains examined were immune to infection by the four O139 phages. If the parent strains are considered to be permanent lysogens, the others may be lysogens which have lost the prophage but retained the immunity phenotype. A similar observation has been reported for phage CP-T $1,{ }^{14}$ the best studied temperature phage of $V$. cholerae. The immunity profile of the 0139 phage resembled that of phage $\alpha$. Unlike phage $\alpha$ lysogens, the $\mathrm{O} 139$ phage lysogens of $V$. cholerae MAK 757 were resistant to phage $\beta$ infection. Thus the new phage may form an immunity class of its own.

Infection of $V$. cholerae MAK 757 with 0139 phage produced turbid plaques characteristic of lysogens. Single colony isolates of these lysogens liberated free phage even after 10 broth subcultures with single colony isolation at each stage. The auxotrophic profiles of these putative lysogens showed that $c .75 \%$ had acquired a requirement for purines. This strongly suggested the integration of the prophage on the host chromosome and confirmed the lysogeny. However, direct confirmation by hybridisation of chromosomal DNA from lysogens with phage DNA has not yet been done. The yield of phage was very low and, despite repeated efforts, the lysogens could not be induced to lyse to produce high-titre phage stock necessary for its 
molecular characterisation. All $V$. cholerae MAK 757 lysogens maintained their parental O1 serotype and the El Tor biotype, as shown by sensitivity to phages $\mathrm{e}_{4}$ and $\mathrm{e}_{5}$.

\section{References}

1. Wilson G. Cholera. In: Smith GR, Easmon CSF (eds) Topley and Wilson's Principles of bacteriology, virology and immunity, 7th edn, vol 3, Bacterial diseases. 1984: 446-457.

2. Morris JG. Non-O group 1 Vibrio cholerae: a look at the epidemiology of an occasional pathogen. Epidemiol Rev 1990; 12: 179-191.

3. Garg S, Saha PK, Ramamurthy T et al. Nationwide prevalence of the new epidemic strain of Vibrio cholerae 0139 in Bengal in India. $J$ Infection $993 ; 27: 108-109$.

4. Shimada T, Nair GB, Deb, BC, Albert MJ, Sack RB, Takeda Y. Outbreaks of Vibrio cholerae non-O1 in India and Bangladesh. Lancet 1993; 341 : 1347.

5. Nair GB, Takeda Y. Vibrio cholerae in disguise - a disturbing entity. World J Microbiol Biotech 1993; 9: 399-400.

6. Das B, Ghosh RK, Sharma C, Vasin N, Ghosh A. Tandem repeats of cholera toxin gene in Vibrio cholerae $\mathrm{O} 139$ Lancet 1993; 342: 1173-1174.

7. Mukerjee S. Principles and practice of typing Vibrio cholerae. Methods Microbiol 1978; 12: 51-115.
This work was supported by grant no. BT/TF/9/63/91 from the Department of Biotechnology, Government of India. S.K. is grateful to the Council of Scientific and Industrial Research for the award of a Junior Research Fellowship. Technical assistance of T. Chaudhuri and R. Shit is gratefully acknowledged.

8. Clowes RC, Hayes W (eds). Experiments in microbial genetics. Oxford, Blackwell Scientific Publications. 1968: 228.

9. Siddiqui KAI, Battacharyya FK. Role of temperate phage in determining lytic phage sensitivity and serotype of Vibrio cholerae. Infect Immun 1982; 37: 847-851.

10. Nicolle P, Gallet J, Schraen M-F, Brault J. Diversité des états lysogènes parmi vibrions cholériques el tor de provenances variées. Bull Soc Pathol Exot Filiales 1971; 64: 603-612.

11. Gerdes JC, Romig WR. Complete and defective bacteriophages of classical Vibrio cholerae: relationship to the Kappa type bacteriophage. J Virol 1975; 15: 1231-1238.

12. Mitra SN. Mutation induced by vibriophage PS166 infection changes biotype and phage type of Vibrio cholerae. $\mathrm{J} \mathrm{Med}$ Microbiol 1989; 30: 137-141.

13. Pajni S, Sharma C, Bhasin N et al. Studies on the genesis of Vibrio cholerae $\mathrm{O} 139$ : identification of progenitor strains. $J$ Med Microbiol 1994; 41 : (in press).

14. Guidolin A, Manning PA. Bacteriophage CP-T1 of Vibrio cholerae. Identification of the cell surface receptor. Eur $J$ Biochem 1985; 153: 89-94. 